\title{
Socio-cultural Approach in Developing the Quality of Clinical Education in the Millennium Era: A Literature Review (New Paradigm)
}

\author{
1.2. Lisnadiyanti, ${ }^{3 .}$ Dr. Moses Glorino Rumambo Pandin M.Si., M.Phil., M.Psi., Psikolog \\ ${ }^{1}$ Program Doktor FKP Universitas Airlangga Surabaya \\ 2.Profesi Ners FKIK Univesitas Binawan \\ ${ }^{3 .}$ Departemen Sastra Inggris, Fakultas Ilmu Budaya, Universitas Airlangga
}

Email: lisnadiyanti-2020@fkp.unai.ac.id

\begin{abstract}
Clinical education is a method that is applied to formal nurse education as a step to provide real and direct learning experiences in the nursing environment correctly and effectively. The success of education in a clinical setting certainly requires the support of teaching nurses (clinical instructors) who have credibility and competence in terms of knowledge, attitudes and skills and are actively involved in professional activities. The diversity of backgrounds of nurses and students, including patients, certainly contributes to a shift in paradigms and perspectives for the nursing environment both in education and in clinical settings in health services. Responding to this cultural diversity, it is important to prepare knowledge and understanding related to transcultural nursing issues, intercultural communication and clinical education which explores the socio-cultural elements in the implementation of staff, students and patients. Purpose: The purpose of this literature review is to identify the extent to which nurse educators play a role by including socio-cultural and transcultural aspects in efforts to develop the quality of education in clinical practice environments in the millennium era. Method: The method of writing this article uses 11 literature review, the publication year period 2019,2020 and 2021 with sources from 4 databases such as science Direct, Scopus, ProQuest and Elsevier. The review guidelines used are based on Prisma and the Joanna Briggs Institutute. The level of eligibility is identified through the title, abstract, research methodology as well as the type of scholarly journal and full text. Results: The results of the reviews found are presented in a narrative form. The results of the review study found that there were 11 articles explaining the competence of clinical education based on the socio-cultural approach, which is an educational strategy in the clinical area that integrates transcultural elements of nursing, intercultural communication, collaboration, self-directed with the principles of openness, honesty, and mutual respect in the implementation of team interaction and collaboration. The development of interpersonal relationships is also an important role that educators must have in helping to introduce the nurse orientation process to the organizational environment and other professional teams so that the achievement of satisfaction with clinical education is able to improve the performance of nurses and students perfectly. Conclusion: Clinical education which is supported by the competence of nurse educators (clinical instructors) who have individual and professional competences has a role to play in improving clinical learning outcomes by both students and nurses with a socio-cultural and transcultural strategic approach that will create satisfaction with the achievement of clinical competence and performance effectively.
\end{abstract}

Keywords: clinical instructor, clinical education, transcultural, intercultural communication, socioculture and millennial generation 


\section{INTRODUCTION}

Nursing as a professional and professional staff with the largest number of service personnel in health services (60\% to $70 \%$ of the composition of the hospital workforce), of course, must be supported by strengthening the quality both at the formal and informal education levels and oriented to the needs of the community according to the development of culture and civilization. Nursing education is education in the applied Sciences category, which is an integration of nursing knowledge, attitudes and skills through real experience in clinical learning(1)(2). The clinical learning process includes the transformation of nursing science, teaching nursing skills and role models to nurse administrators, especially beginners who requires good, comfortable interpersonal relationship competences and creates mutual respect and trust and satisfaction of the learning process which has an impact on the quality of learning outcomes for student nurse or new nurse(3). A nurse educator is a nurse who has a nurse qualification with the ability of the arts and sciences of nursing and becomes a liaison between students or new nurses and the health service environment so as to provide opportunities for clinical practice adaptation and opportunities for meaningful clinical orientation without stress, conflict and pressure on learners(4).

Mentoring in the clinical learning process, especially for novice nurses, requires effective communication skills to be able to carry out collaborative tasks together with a team of nurses and other health workers, especially between clinical instructors (nurse educator) and new nurses so that the presence of new nurses is able to adapt with other health teams that allow a conducive atmosphere and minimize stress and interpersonal conflicts for generally novice nurses who are at the orientation stage(5)(6). Strategies for building and building effective relationships with role models are important to be learnt and understood(7).

Communication is a way to exchange information or a means in the process of transforming knowledge and skills for nurses, especially for nurses in the orientation and entry stages to improve basic clinical skills in their respective work units(8). In a clinical setting, the role of clinical instructors is needed to bridge theory, practice and real situations in the field as well as the implementation suitability of the 3 elements, moreover the formation of a conducive atmosphere and environment as well as good leadership support, healthy, safe and comfortable work units have an impact which is significant for the clinical learning process for novice nurses(9)(10). Many countries use mentoring by clinical nursing staff who play an important role as preceptors or facilitators in developing continuous clinical practice learning starting from learning preparation in the classroom, in simulations and clinical practice in the laboratory based on previous learning laboratory experiences in a variety of backgrounds(11)(12)(13). It is really important to build harmonious interpersonal relationships and impact on satisfying clinical experiences between the perceiver and novice nurses in an effort to overcome various psychological pressures, anxiety, that leads to frustration and failure in the clinical learning process(14)(15). In line with the development of nursing science in the 21 st century with Era 4.0 to 5.0, the advances in health and information technology must be addressed by nursing and it is 
necessary to pay attention to the emergence of millennial generations and cultures in the nursing community both in education and in clinical practice environments(16)(17).

Subsequent studies conducted by Collier synthesized 37 articles related to the elements of clinical nurse competence (Clinical Instructure) including: the ability to develop interpersonal relationships, nursing competence and clarity of attitudes(18). The development of interpersonal relationships is a major component and the largest contribution in the global clinical community for a nurse educator (clinical instructor). Based on the results of the synthesis of articles conducted by Collier it was revealed that in the past 15 years the element of nursing competence was a priority for teaching nurses, but for the current condition, the greatest ability of teaching nurses is the competence of developing interpersonal relationship skills(18). However, the attitude also needs to be formed in nurse educator in the presence of elements: approachability, fairness, openness, empathy, honesty and mutual respect in diversity of backgrounds(18). The development of technology and community culture of health in the millennium era certainly has implications on the acceleration of the significant influence on changes in the characteristics of nurses and students and organizations, considering that the development of health and communication technology encourages changes in orientation and innovation in the clinical education environment and the application of models in the development of four-oriented clinical learning process strategies (innovative Teaching Model):

1. The Problem - Based Leaning method in nursing education, (PBL).

2. The Self - Regulated Learning strategy, (SRL).

3. The developing nursing students' reflective skills associated as a key component in the perceived coherence between theory and practice.

4. The computer - based clinical simulation(19).

\section{METHOD}

The use of the method in this literature study is based on a review of 30 literatures on the scope of clinical nursing education from 2019 to 2021 that is relevant to the topic and then it is strictly selected so that it has closer closeness to the topic issues discussed.

\section{LITERATURE STUDY PROTOCOL}

Creating a protocol with the PRISMA (Preferred Reporting Items for Systematic Reviews and Meta-analyzes) concept approach with the JBI CRITICAL APPRAISAL CHECKLIST FOR assessment guide with the following steps: 1. compilation of inclusion criteria as a standard of eligibility for literature (original research articles), 2. literature search on the online database which has a reputation of Q1, Q2, and Q3 on Scopus, Science direct, ProQues, Elsevier and according to the inclusion criteria, 3. Selection of literature by keyword, abstract or partial from journals and topic selection with "AND" and "OR", 4 . collection of data or information related to journals (year, topic, country, author, methodology, type of article .... etc.)

\section{ARTICLE SEARCH STRATEGY}


Articles were obtained by searching on 4 electronic databases, namely Scopus, Science Direct, and ProQuest Journal and Elsevier. Keywords used according to the chosen theme are Clinical education, Clinical Educator, Social Culture, Intercultural Communication, Nurse Educator, Experiential theory.

\section{INCLUSION CRITERIA}

The criteria for the inclusion of articles regarding Clinical Practice and social cultural approaches are determined based on the following: journal topics published in 2019 to 2021, Literature accessed using English, full text access, Quantitative and qualitative research designs or mix methods with sample populations and variables related to clinical practice and social cultural approach. Guidelines for reviewing these database journals are using the Joanna Briggs Institute (JBI) assessment tool and the PRISMA guide, with eligibility study from the title, abstract, methodology, results and full text and type of scholarly journal sources.

\section{RESULTS}

Reviews contained in 15 journals regarding Clinical Development Practice and social cultural approaches from the inclusion criteria of quantitative and qualitative research designs from 3 electronic searches of databases, namely Scopus, Science direct and ProQues, obtained the following results:

1. Journal Title: Characteristics of an Effective Nursing Clinical Instructor: The State of the Science.(18)

This journal writing is motivated by the importance of the role of clinical instructors in the development process of clinical education in every health service setting, in terms of the competency characteristics of interpersonal relationships and the traits that a clinical instructor must be able to see whether the role is being carried out effectively or not. Furthermore, analyzing the characteristics of perceptions of the effectiveness of teaching nurses (clinical instructor nurses / CI) by measuring the level of effectiveness. (from the point of view and perception of student nurses). The results in the findings of this journal indicate that there are three elements that most dominate in seeing the effectiveness of the important role of a clinical instructor, namely:

- The ability to demonstrate clinical skills.

- The ability to develop interpersonal relationships.

- Having a character and positive traits that are clear, honest and open.

2. Journal title: Undergraduate nursing students' perspectives of intercultural communication: A qualitative descriptive study.(20)

The background of this research begins with the findings of various changes in health service organizations in line with community demands for increasingly multi complex health services so that they have a direct impact on professional practice, providing patient-centered services, implementing a safe culture, cultural elements that affect 
communication from nurse to patient who must receive attention seriously since the education stage. This study was conducted to see the experiences and perceptions of nursing students in intercultural communication practices. The findings in this study indicate that there are limitations to the substance of the curriculum regarding intercultural communication that are felt by all nurse participants based on participants' perceptions that intercultural communication has a strong relationship in facilitating the growth of empathy and culture-based nursing practice with the principle of prioritizing safety for patients. The barriers to intercultural communication practice consist of the following elements:

- Language

- Gender.

- Level of authority (power).

- The limited number of nurses.

However, cultural background, previous practical experience, exposure to social media and learning to understand cultural diversity in the environment can facilitate interpersonal communication competence. For this reason, it needs a development that integrates cultural elements in the health service protocol in an active learning strategy to equip student nurses so that they have the expertise to understand and carry out intercultural communication effectively.

3. Journal title: Nurse trainees' perception of effective clinical instructor characteristics. (21)

This study has the background of the emergence of gaps from various elements of clinical instructor competence including clinical education and previous clinical experience which play an important role in shaping the quality of clinical education and encouraging the effectiveness of clinical practice with the aim of improving the quality of patient services. The findings of this study indicate that the level of effectiveness Clinical instructor nurses as trainees who attended the training program for less than 6 months had better scores than nurses who were trained for more than 12 months. Statistically, it shows the results of teaching ability ( $\mathrm{P}$ 0.005), nursing competence ( $\mathrm{P}$ 0.040), and interpersonal relations $(\mathrm{P}$ 0.007) and does not show a significant difference in the evaluation component or personal characteristics of clinical instructors. Overall, the findings with the highest score were on the personality component $(84 \%)$ and relatively low on the teaching ability competency $(81.3 \%)$. Almost all trainees have the same view of the effectiveness as measured by the rating on each dimension of the clinical instructor's ability.

4. Journal Title: Interpersonal skills mediate the relationship between communicative and clinical competencies among nursing students: A descriptive study.(15)

This study is based on the development of a phenomenon in the area of nursing clinical practice that is able to create an atmosphere that does not trigger stress, the high number 
of nurses in and out of nurses and an increase in clinical practice satisfaction and academic performance. There are still many complaints that indicate the low level of communication and clinical competence for newly graduated nurses and there are no studies that analyze the relationship between interpersonal skills and these two components. The findings of this study indicate that female students in the 4th year study were satisfied with the learning outcomes and clinical practice at a more advanced skill competency level. There are five categories of highest scores in sequence, consisting of:

- Organizational understanding.

- Data collection.

- Overcoming stereotypes.

- Cooperative.

- Interpersonal skills.

In addition, the findings on the competence of interpersonal skills comprehensively become a supporting factor that connects elements of communicative competency on clinical competency (explanatory power $=53.8 \%$ ). The conclusion from the final findings in this study shows that clinical competence has a meaningful relationship to communication competence $(\mathrm{r}=0.52, \mathrm{p}<.001)$ and interpersonal skills $(\mathrm{r}=0.72, \mathrm{p}$ $<.001$ ), then there is a significant relationship also on communication competence towards interpersonal skills $(r=0.64, \mathrm{p}<.001$; ). The limitation of this study is that it does not identify students' perceptions based on the study period so that it describes the facts of the phenomena found more specifically based on the length of the study and the level of exposure in the clinical practice area.

5. Journal titles 5: Language-specific skills in intercultural healthcare communication: Comparing perceived preparedness and skills in nurses' first and second languages.(22) Journal titles 6: Overseas qualified nurses' sociocultural adaptation into the Australian healthcare system: A cross-sectional study.(23)

The background of these two studies is based on the dynamics in the process of interaction that occurs in individuals with diverse cultural backgrounds and encourages improvements and changes in the arrangement of health services. The ability of each individual to adapt to an environment characterized by various adaptation difficulties so that there is a need for intelligence and competence of nurses to learn and manage cultural elements through understanding the characteristics of the dominant nurse-patient interaction with cultural and linguistic diversity, it is increasingly felt that nurses' readiness and special skills are needed to provide cross-cultural based services starting with the understanding and use of the main language of instruction and the second language. The relationship between socio-cultural elements has an influence on increasing the competence, satisfaction and comfort of nurses in line with the adaptation process to the work environment in any clinical condition. Thus, it is necessary to 
strengthen the socio-cultural aspects of teaching nurses. The results of this study explain the number of nurses who have significantly low self-confidence when carrying out nursing practices with the necessity to use a second language of instruction as a form of cross-cultural implementation so that it is necessary to have special training that encourages the development and improvement of special communication skills based on the second language of instruction. The limitation of this study is only based on one characteristic of the geographic area of nurses and does not involve elements of other health workers so that the level of representation is very limited.

6. The 7th Journal Title: Clinical mentors' experiences of their intercultural communication competence in mentoring culturally and linguistically diverse nursing students: A qualitative study (8th journal on LR).(9)

This study is motivated by the issue of intercultural communication which is increasing and encouraging change as a result of high cross-cultural mobility for both patients, health professionals and student nurses. The development of cultural issues and problems in the health care environment makes it a challenge for all health professionals, including nurses, especially nursing educators in the clinic area. The challenge of cultural diversity is an encouraging factor to prepare clinical educators and student nurses to form competences professionally in intercultural communication skills. The findings of this study explain the experiences and statements of clinical mentors in an effort to build empathy character in increasing motivation to develop intercultural communication competencies. So far, limited resources and superiors' support have been perceived as obstacles both psychologically and ethically which have contributed to the decline in motivation of the mentors. Mentors often experience fear due to cultural uncertainty, but this can be controlled through positive experiences during the mentoring process and interactions by discussing cultural elements directly. The conclusion is that knowledge about intercultural communication competence can be achieved effectively if supported by a clinical mentor, the expertise of a mentor to provide benefits and success of services in the clinical area for staff, patients and student nurses.

7. The 8th Journal Title: Clinical Instructor Social Support and Nursing Student Stress in Clinical Environments.(24)

The complexity of the dynamic health service environment and a new challenge in the clinical practice area certainly provides a lot of input that must be responded positively to nursing practitioners both staff and student nurses and implemented starting from the level of nursing education. Various elements that cover environmental aspects in a clinical setting include the physical and social environment which underlies the growing need for learning in clinical practice that is free from high potential stress. The phenomenon that often appears in the clinical practice area is the low level of social support, which in this case is the nursing staff as clinical instructors and is related to the 
emergence of stress faced by students who will carry out clinical learning in every setting of health services. This study resulted in findings, among others, that all participants received $76.5 \%$ moderate social support and $12.1 \%$ high stress levels with a stress level of $65.5 \%$ at moderate stress levels due to academic assignments in the clinic area. In general, it can be stated that the study findings showed a significant correlation between perceptions of social support and the age of the participants, the level of study period and the level of stress in students $(\mathrm{P}<0.001)$. The conclusion from the findings of this study is to emphasize the importance of understanding social support starting from the implementation of learning on campus, especially training programs for all teachers to recognize and understand the importance of social support in socialization programs to become reliable professionals.

8. Journal Title: Characteristics of a supportive clinical instructor in nursing training: A qualitative study.(25)

This study is motivated by the high workload of nurses and the level of tight supervision from various superiors which results in increased stress for nurses and the emergence of different behavioral responses. This condition is of course the basis for controlling a conducive clinical atmosphere through training programs with the support of nurse instructors who are able to assist in a conducive manner with a cooperative, communicative and supportive attitude and are able to give nurses the opportunity to understand their duties, policies and organization. The findings in this study explain the two main characteristics of a supportive clinical instructor, namely individual competency components (patient, calm, polite and able to control oneself) and professional competence (knowledge, skills, effective communication, role, positive attitude, equality with nurses/ student nurses). Thus, the role of a clinical instructor can encourage increased learning and training outcomes so as to produce good and effective quality skills.

9. Journal Title: Applying Newman's Theory of Health Expansion to Bridge the Gap between Nursing Faculty and Generation Z.(26)

The background of this study begins with the emergence of a millennial civilization and culture which is dominated by the younger generation of the digital era known as generation $\mathrm{Z}$. The character of this generation group is unique and is present to fill the health service structure including nursing education institutions. The phenomenon of generation $\mathrm{Z}$ is a significant challenge for the perspective of the world of Nursing education to the presence of this generation who since the beginning of their lives have known technology well. The presence of this millennial generation is the background for a paradigm shift and approach to the world of nurse education through attitude strategies and role characters that show more closeness or familiarity, more effective learning, being able to survive in a situation and exploring the ideology of generation $\mathrm{Z}$ groups 
towards the meaning of success for them. Newman's theory clarifies how learning strategies for critical thinking in generation $\mathrm{Z}$ groups through finding meaning in real life experiences. Following up on this theory, nursing education institutions must provide opportunities for students to determine expected learning goals through direct clinical learning experiences (clinical practice) and social learning through team-based learning methods and case studies. The conclusions that can be formulated in this study are that generation $\mathrm{z}$ has very significant differences characterized by collective life experiences, traditions, beliefs and models of care and has a relationship to forms and learning strategies that prioritize creativity and innovation.

\section{Journal title: Social- and Health Care Educators' Cultural Competence. (27)}

The background of this study is based on changes in the demographic conditions of society and global cultural civilization, the multicultural background of nurses that has led to changes in social and education in health services. Responding to this, of course, the interested parties provide an educational strategy with a load of cultural competences and prepare nurse educators (clinical instructors) with synergistic competences on transcultural education. The findings of this study explain that the cultural competence of teaching nurses in the clinic is transcultural education with the categories: educatorship and ethical attitudes, including elements of language, characteristics of learning methods and styles, integrated multicultural nurses, knowledge and cultural sensitivity, collaboration, self- awareness and openness, respect and care as well as giving protection. It can be concluded that cultural competence is representative of the main competencies of educators in health services. Nurse educators must really understand and know the background of nurses or student nurses and the procedures for a more flexible learning model with a pedagogical approach and have ethical principles and openness.

\section{Journal title: The challenges of supporting nursing students in clinical education}

This study has a background in the development of a clinical environment that requires the formation of an atmosphere that supports the learning of both students, orientation period nurses who need to be supported by the preparation of competency and nursing expertise as a challenge for the nurse profession in the future. Clinical education is a very fundamental learning strategy as a basis for developing nursing professional skills by providing opportunities for new students and nurses to transform knowledge in concepts and skills and expertise in providing nursing services to patients. This phenomenon creates a big challenge in the effort to create clinical learning strategies that support the goals and outcomes of clinical practice in students. The most dominant form of challenge that often occurs in a clinical environment where students are placed consists of: tough and hostile attitudes, fear, anxiety, socio-cultural challenges, stress originating from the organizational environment in a clinical setting as a poor adaptation process, the emergence of discriminatory attitudes in nurse students and medical profession students 
which lead to assumptions of the absence of good support and the ineffectiveness of the learning process in clinical practice. The findings in this study indicate the magnitude of the challenges that must be faced by nursing students and nurses who have just entered the health service environment, who are required to be able to face and adapt to various dynamic and very multi-complex conditions as challenges in the clinical environment. Therefore, it needs the manager and nurse educator's competence in a clinical practice environment and understands the priorities needed in the learning process and how to achieve clinical competence which is formulated in a practical learning design in a supportive and effective manner. The success of clinical education will certainly show the good quality of clinical education through learning outcomes and professional cultural justification.

12. Journal Title: Nursing education in the path of globalization: Promotion or challenge?

The background of this study is based on the rise of a civilization and culture that attracts the attention of all people from various layers of society as well as various scientific disciplines and influences all aspects of life such as social, cultural, political, economic and shifting to the health service sector as a significant challenge. In this study there are nine categories that are analyzed to have the impact of the globalization era on nursing, namely: the development of the nursing profession at the global level, mobility and movement of nurses from and out, exchange of information and interactions in nursing, higher education institutions of nursing, professional authority, specialization nursing, professional ethics, management and supervision as well as professional independence. The existence of a very intensive globalization influence on the nursing profession does not always have the same consequences in all social sectors, including the process of social, cultural and technological adaptations, all of which are very important to be addressed appropriately and effectively. The civilization and culture of globalization is a phase of development that cannot be avoided due to various advances and improvements in the nursing profession globally and creates its own challenges in the nursing profession, especially related to the mobility and transfer of nurses as well as various ethical issues. Globalization also has implications for broader needs and is not limited to knowledge about caring but must also be supported by competence in intercultural communication and the use of technology in more intensive daily activities. The globalization of health and nursing services characterizes the absence of boundaries or striking differences between regions of the country as evidenced by the agreement on integrated international nursing service standards used as a reference for nursing services in all countries. Globalization nursing provides nurses the opportunity to learn and understand broadly about the application of health care standards in various countries including beliefs, culture, rituals and ideas which all have varying degrees of difference. Globalization is also shown by the large number of nurses who have moved abroad to get 
jobs such as England, Australia and New Zealand, of course through the support of information and communication technology. The progress of the globalization civilization is also felt in the nurse-patient relationship which makes it easier to access various information and resources needed to meet the needs of patients quickly and effectively.

\section{Journal Title: Nursing Lecturers' Perception and Experience of Teaching Cultural} Competence: A European Qualitative Study

The background of this study is based on cultural competence which is an important substance in providing health services effectively and based on a cultural approach as a form of service responsiveness, reducing service inequality, challenges of racism in service delivery and increasing patient safety as well as satisfaction and the end result of service quality itself. This condition makes the reason for student nurses and new nurses to be able to understand and be competent in terms of the ability to develop competences culturally both through special training and professional education. This study aims to investigate the perceptions and experiences of teaching staff in providing knowledge and understanding of cultural-based competencies during the higher education process of nursing. This study uses a selective phenomenological approach so as to avoid the tendency of illegitimate perceptions and experiences to emerge during the process of knowledge transformation of cultural competence.

Nursing services should lead to social, cultural and biological dimensions in a balanced and appropriate manner to all individuals. The quality of nursing services with a crosscultural approach eliminates discriminatory attitudes in all factors that affect the quality of their health. Understanding of cultural values, beliefs and practices is the main basic element as a form of attitude awareness in adjusting cultural values in an effort to provide effective and useful nursing services. Cultural competence in nursing shows that nursing service is holistic by appreciating the differences and similarities in cultural values, beliefs and lifestyles so that it provides opportunities for nurses to be more aware of cultural values, competence and fair attention.

14. Journal Title: Educators' perceptions and views of problem-based learning through simulation

This study has a background from the emergence of various problems and the many changes in the environment that provide new challenges to the development of nursing education recently and must be treated in a balanced manner between the education environment and health services through a learning strategy through a problem-based learning approach with a simulation strategy. The emergence of various health problems and diseases in the community such as tuberculosis, HIV-AIDS and Covid-19 encourages 
the use, regulations and policies of the latest health technology. The learning approach through problem-based learning that is oriented towards student-centered learning certainly gives students the opportunity to be actively involved in learning situations in the clinical practice area and get a real picture of the conditions in the clinic and be able to apply and analyze the gaps between knowledge and practice. The purpose of this study is to explore the extent to which a problem-based learning approach can be used and to facilitate learning strategies using simulation methods. The findings in this study indicate that the problem-based learning approach through the simulation method is able to create attitudes and behaviors in teamwork and is able to demonstrate a holistic learning process in an effort to take action and nursing care. The final conclusion that can be explained in this study is that problem-based learning through simulation methods can be applied to form changes in knowledge in students who are more innovative, creative, and able to develop the ability to think critically in analyzing increasingly complex health problems.

\section{CONCLUSION}

The clinical learning process includes the transformation of nursing science, teaching nursing skills and role models to nurse administrators, especially beginners who requires good, comfortable interpersonal relationship competences and creates mutual respect and trust and satisfaction of the learning process which has an impact on the quality of learning outcomes for student nurse or new nurse. Mentoring in the clinical learning process, especially for novice nurses, requires effective communication skills to be able to carry out collaborative tasks together with a team of nurses and other health workers, especially between clinical instructors (nurse educator) and new nurses so that the presence of new nurses is able to adapt with other health teams that allow a conducive atmosphere and minimize stress and interpersonal conflicts for generally novice nurses who are at the orientation stage. Clinical education which is supported by the competence of nurse educators (clinical instructors) who have individual and professional competences has a role to play in improving clinical learning outcomes by both students and nurses with a socio-cultural and transcultural strategic approach that will create satisfaction with the achievement of clinical competence and performance effectively.

\section{REFERENCE}

1. Chen B, Wang Y, Xiao L, Xu C, Shen Y, Qin Q, et al. Effects of mobile learning for nursing students in clinical education: A meta-analysis [Internet]. Vol. 97, Nurse Education Today. Elsevier Ltd; 2021. 104706 p. Available from: https://doi.org/10.1016/j.nedt.2020.104706 
2. Lee YH, Lin SC, Wang PY, Lin MH. Objective structural clinical examination for evaluating learning efficacy of Cultural Competence Cultivation Programme for nurses. BMC Nurs. 2020;19(1):1-8.

3. Tuohy D. Effective intercultural communication in nursing. Nurs Stand. 2019;34(2):4550 .

4. Antón-Solanas I, Huércanos-Esparza I, Hamam-Alcober N, Vanceulebroeck V, Dehaes S, Kalkan I, et al. Nursing lecturers' perception and experience of teaching cultural competence: a european qualitative study. Int J Environ Res Public Health. 2021;18(3):122.

5. Bhurtun HD, Azimirad M, Saaranen T, Turunen H. Stress and coping among nursing students during clinical training: An integrative review. J Nurs Educ. 2019;58(5):266-72.

6. Cornine A. Reducing Nursing Student Anxiety in the Clinical Setting: An Integrative Review. Nurs Educ Perspect. 2020;41(4):229-34.

7. Molina-Mula J, Gallo-Estrada J. Impact of Nurse-Patient Relationship on Quality of Care and Patient Autonomy in Decision-Making. Int $J$ Environ Res Public Heal. 2020;17(835):1-24.

8. Gutiérrez-Puertas L, Márquez-Hernández V V., Gutiérrez-Puertas V, Granados-Gámez G, Aguilera-Manrique G. Educational Interventions for Nursing Students to Develop Communication Skills with Patients: A Systematic Review. Int J Environ Res Public Heal. 2020;17(2241):1-21.

9. Hagqvist P, Oikarainen A, Tuomikoski AM, Juntunen J, Mikkonen K. Clinical mentors' experiences of their intercultural communication competence in mentoring culturally and linguistically diverse nursing students: A qualitative study. Nurse Educ Today [Internet]. 2020;87(December 2019):104348. Available from: https://doi.org/10.1016/j.nedt. 2020.104348

10. Filmer T, Herbig B. A training intervention for home care nurses in cross-cultural communication: An evaluation study of changes in attitudes, knowledge and behaviour. $\mathrm{J}$ Adv Nurs. 2020;76(1):147-62.

11. Koukourikos K, Tsaloglidou A, Kourkouta L, Papathanasiou I V, Iliadis C, Fratzana A, et al. Simulation in Clinical Nursing Education. ACTA Inf MED. 2021;29(1):15-20.

12. Hsieh P-L, Chen S-H. Effectiveness of an Evidence-Based Practice Educational Intervention among School Nurses. Int J Environ Res Public Heal. 2020;17(4063):1-10.

13. Burgess A, van Diggele C, Roberts C, Mellis C. Key tips for teaching in the clinical setting. BMC Med Educ [Internet]. 2020;20(Suppl 2):1-7. Available from: http://dx.doi.org/10.1186/s12909-020-02283-2

14. Alammar K, Ahmad M, Salem O, Almutairi S. Nursing Students' Perception of the Clinical Learning Environment. Open Nurs J. 2020;14(3). 
15. Kang K, Lee M, Cho H. Interpersonal Skills Mediate the Relationship Between Communicative and Clinical Competencies Among Nursing Students: a Descriptive Study. Nurse Educ Today [Internet]. 2021;99(November 2020):104793. Available from: https://doi.org/10.1016/j.nedt.2021.104793

16. Abbas S, Zakar R, Fischer F. Qualitative study of socio-cultural challenges in the nursing profession in Pakistan. BMC Nurs. 2020;19(1):1-7.

17. Korkis L, Ternavan K, Ladak A, Maines M, Ribeiro D, Hickey S. Mentoring Clinical Nurses Toward a Just Culture. J Nurs Adm. 2019;49(7/8):384-8.

18. Collier AD. Characteristics of an effective nursing clinical instructor: The state of the science. J Clin Nurs. 2017;27(1-2):363-74.

19. Li P, Li C. A Four-in-one Teaching Model for Fundamental Nursing Based on UCD Theory. Int J Emerg Technol Learn. 2020;15(15):188-201.

20. Armah N, Martin D, Harder N, Deer F. Undergraduate nursing students' perspectives of intercultural communication: A qualitative descriptive study. Nurse Educ Today [Internet]. 2020;95(August):104604. Available from: https://doi.org/10.1016/j.nedt.2020. 104604

21. Hababeh MO, Lalithabai DS. Nurse trainees' perception of effective clinical instructor characteristics. Int J Nurs Sci [Internet]. 2020;7(3):285-90. Available from: https://doi.org/10.1016/j.ijnss.2020.06.006

22. Gasiorek J, van de Poel K. Language-specific skills in intercultural healthcare communication: Comparing perceived preparedness and skills in nurses' first and second languages. Nurse Educ Today [Internet]. 2018;61(November 2017):54-9. Available from: https://doi.org/10.1016/j.nedt.2017.11.008

23. Zanjani ME, Ziaian T, Ullrich S, Fooladi E. Overseas qualified nurses' sociocultural adaptation into the Australian healthcare system: A cross-sectional study. Collegian [Internet]. 2021;(xxxx). Available from: https://doi.org/10.1016/j.colegn.2020.12.005

24. Far MS, Khah HZH, Moradbeigi K, Hatefi-Moadab N, Ghassemi M, Cheraghian B, et al. Clinical Instructor Social Support and Nursing Student Stress in Clinical Environments. Int J Adv Biotechnol Res. 2017;8(1):182-8.

25. Farahani MA, Joolaee S, Zabihi A, Varaei S. Characteristics of a supportive clinical instructor in nursing training: A qualitative study. J Nurs Midwifery Sci. 2020;7(4):22632

. 26. Antón-Solanas I, Huércanos-Esparza I, Hamam-Alcober N, Vanceulebroeck V, Dehaes S, Kalkan I, et al. Nursing lecturers' perception and experience of teaching cultural competence: a european qualitative study. Int J Environ Res Public Health. 2021;18(3):122. 
27. Edwards-Maddox S, Cartwright A, Quintana D, Contreras JA. Applying Newman'S Theory of Health Expansion To Bridge the Gap Between Nursing Faculty and Generation Z. J Prof Nurs [Internet]. 2021; Available from: https://doi.org/10.1016/j.profnurs. 2021.02.002

28. Han R, Koskinen M, Mikkonen K, Sjogren T, Korp H, Koivula M, et al. Social- and Health Care Educators' Cultural Competence. Int J Caring Sci [Internet]. 2020;13(3):1555-62. Available from: http://www.internationaljournalofcaringsciences. org/docs/3_koskinen_original_13_3_2.pdf

29. Sharifi N, Adib-Hajbaghery M, Najafi M. Cultural competence in nursing: A concept analysis. International Journal of Nursing Studies [Internet]. 2019 [cited 11 April 2021];99:103386. Available from: https://pubmed.ncbi.nlm.nih.gov/31404821/

30. Matlala S. Educators' perceptions and views of problem-based learning through simulation. Curationis. 2021;44(1):1-8. 